\title{
Evidence of complete band gap and resonances in a plate with periodic stubbed surface
}

\author{
Tsung-Tsong Wu, ${ }^{\text {a) }}$ Zi-Gui Huang, Tzu-Chin Tsai, and Tzung-Chen Wu \\ Institute of Applied Mechanics, National Taiwan University, Taipei 106, Taiwan
}

(Received 28 May 2008; accepted 26 July 2008; published online 16 September 2008)

\begin{abstract}
In this paper, we numerically and experimentally demonstrate the existence of complete band gaps and resonances in a plate with a periodic stubbed surface. Numerical results show that a complete band gap forms as the stub height reaches about three times the plate thickness. In the experiment, we used a pulsed laser to generate broadband elastic waves and used optical devices as well as point piezoelectric transducers to detect wave signals. The results show that the numerical predictions are in very good agreement with those measured experimentally. Remarkable resonances on the top surface of the stubs are found and discussed. (C) 2008 American Institute of Physics. [DOI: 10.1063/1.2970992]
\end{abstract}

Formation of phononic crystal band gaps in a system with periodic arrangement of different elastic properties has been investigated extensively for both bulk ${ }^{1,2}$ and surface ${ }^{3-5}$ waves over the last decade. Increasing attention has been paid to the Lamb ${ }^{6}$ waves due to the coupling of the longitudinal and transversal vibrations, which makes the elastic wave propagation in a thin plate more complicated.

Considering phononic crystal structure in plates, Vasseur et $a l^{7}$ analyzed the band structure of solid/solid twodimensional (2D) phononic crystal plates using the finite element (FE) method. Hsiao et al. ${ }^{8}$ studied experimentally the band structure of steel/epoxy 2D phononic crystal plates and was found to be in good agreement with numerical predictions. However, waves propagating in epoxy plates exhibited strong attenuation and were observed by Vasseur et al. ${ }^{9} \mathrm{Re}-$ cently, plates with 2D phononic film on the surface ${ }^{10,11}$ have received much attention. These structures may generate mini-stop-bands, but not enough for forming a complete band gap.

Different from the previous studies, we analyze the dispersion relation of Lamb waves in a thin plate with stubbed surface both numerically and experimentally using the FE method (COMSOL MULTIPHYSICS ${ }^{12}$ ) and the laser ultrasonic technique, respectively. On one side of a thin aluminum ${ }^{13}$ plate (thickness $h_{1}=1 \mathrm{~mm}$ ) specimen, periodically stubbed cylinders are arranged in a square lattice with lattice constant $\mathbf{a}=10 \mathrm{~mm}$. The diameter of the cylindrical stubs, which are also made of aluminum, is $7 \mathrm{~mm}$ (i.e., $f=0.385, h_{1} / \mathbf{a}=0.1$ ).

To understand the influence of the stubs' height $\left(h_{2}\right)$ on the dispersion, we have conducted a series of numerical calculations. Figure 1 shows the dispersion relations of the thin plate with stubs of different heights for waves propagating along the $\Gamma X$ direction. Upon comparison with the dispersion relation of a homogeneous thin plate [Fig. 1(a)], formation of the special coupling modes can be identified. For example, in Fig. 1(b), when the stub height $h_{2}=0.25 h_{1}$, the purple line marked with $S 0+A 0$ means the mode is transformed from the $S_{0}$ mode and $A_{0}$ modes shown in Fig. 1(a). Similarly, the yellow line marked with $A 0+S 0+A 1$ in Fig. 1(b) denotes the mode is transformed from the $A_{0}, S_{0}$, and $A_{1}$ modes. The

\footnotetext{
a) Author to whom correspondence should be addressed. Electronic mail: wutt@ndt.iam.ntu.edu.tw.
}

evolution of the coupling modes as well as formation of level repulsions ${ }^{14}$ can be identified by comparing Figs. 1(a)-1(c).

In the numerical study, we increase the height of the stubs from one quarter of the plate thickness (with plate thickness denoted as $h_{1}$ ) gradually up to $10 h_{1}$. As the height of stubs increase, some resonances are formed, which result in slower wave velocity and flatter modes ${ }^{15}$ near the edge of the Brillouin zone. We find that as the stub height approaches about triple the plate thickness, i.e., $3 h_{1}$, a narrow complete band gap ranging from 167.5 to $171 \mathrm{kHz}$ forms. Moreover, when the stub height equals $9 h_{1}$, the complete band gap is the largest, with a range from 119 to $157.5 \mathrm{kHz}$ and a relative band gap width equal to 0.278 . When the stub height is ten times the thickness of the plate, i.e., $10 h_{1}$, a secondary large complete band gap appeared, ranging from 119 to $143 \mathrm{kHz}$ (blue region), and three partial band gaps (green region) also appeared, as shown in Fig. 1(d) and Fig. 2.

On the experimental side, we have conducted a series of laser ultrasonic experiments to demonstrate the complete band gap and resonances of the phononic thin plate. The experimental schematic diagram is shown in Fig. 3. The phononic plate with a periodic stubbed surface consisted of

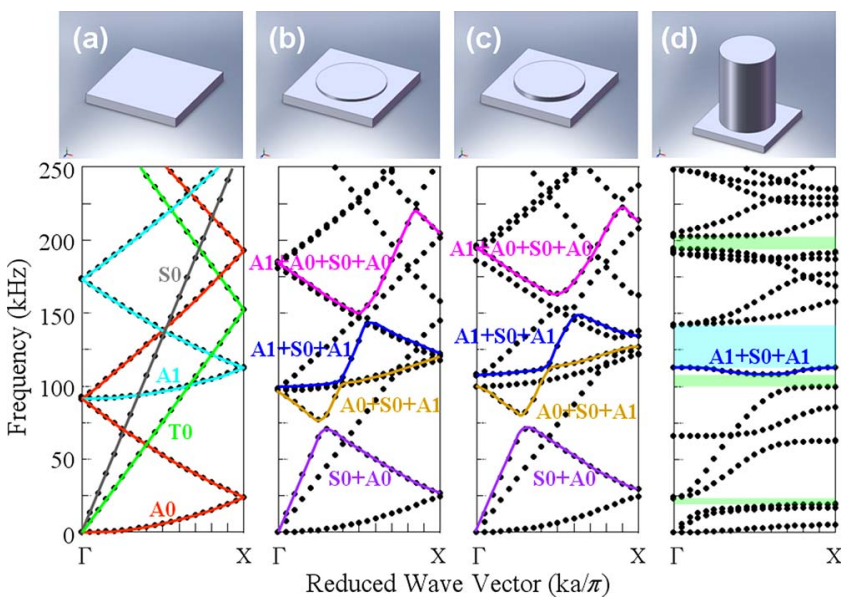

FIG. 1. (Color online) Dispersion relations of the phononic thin plate with stubs of differing height along $\Gamma X$ direction: (a) flat thin plate, (b) stub height $h_{2}=0.25 h_{1}$, (c) stub height $h_{2}=0.5 h_{1}$, and (d) stub height $h_{2}=10 h_{1}$. Lattice constant $a=10 \mathrm{~mm}$, plate thickness $h_{1}=1 \mathrm{~mm}$, and diameter of the cylindrical stub is $7 \mathrm{~mm}$. 


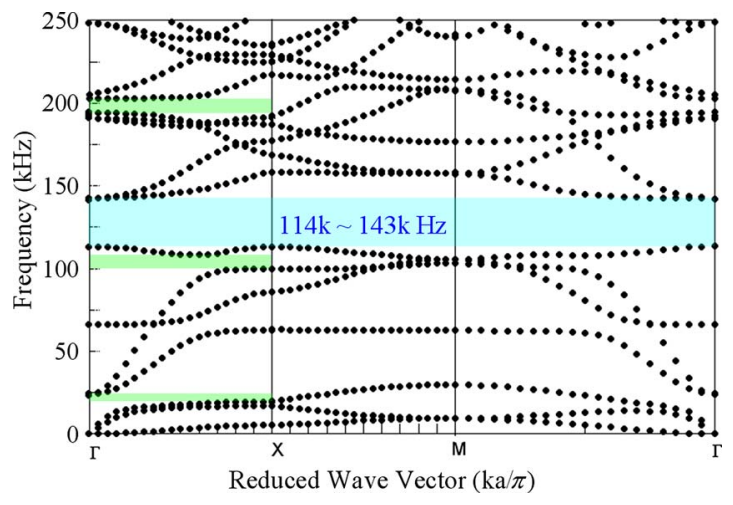

FIG. 2. (Color online) Dispersion relations of the thin plate with a periodic stubbed surface in a square array made of aluminum 6061 with $a=10 \mathrm{~mm}$, $h_{1}=1 \mathrm{~mm}, h_{2}=10 h_{1}$, and $d=7 \mathrm{~mm}$. Complete band gap and partial band gap along the $\Gamma X$ direction are shown as the blue region and green region, respectively.

$16 \times 10$ stubs on one side of the plate. In the experiments, we utilized an Nd:YAG (yttrium aluminum garnet) pulsed laser to generate broadband elastic waves ${ }^{16}$ and an optical interferometer (with He-Ne laser) to measure the normal component of the displacements four rows apart from the laser source. Both the $\Gamma X$ direction and the $\Gamma M$ direction were considered. In the measurements, the digitalized rate of the interferometer was $50 \mathrm{MHz}$.

Shown on the left-hand side of Figs. 4(a) and 4(b) are the measured reference spectra. The reference spectrum is defined as the ratio of the spectrum measured on the phononic thin plate to that measured on a thin flat plate with the same thickness $(1 \mathrm{~mm})$. The right-hand side of Figs. 4(a) and 4(b) show the computed dispersion relations for waves propagating along the $\Gamma X$ and $\Gamma M$ directions, respectively. In Fig. 4(a), the blue region shows the location of the complete band gap, from 114 to $145 \mathrm{kHz}$, which agrees well with the numerical result. The green regions are the partial band gaps along the $\Gamma X$ direction, indicating conformity to the locations and widths of the band gaps. We note that there are some peaks in the reference spectrum around the band edges. For example, the peak around $109 \mathrm{kHz}$ has a value of 1.46 , which means the normal displacement is about one and a half times larger than that of a flat thin plate.

For wave propagating along the $\Gamma M$ direction [Fig. 4(b)], the location and width of the complete band gap also exhibit very good agreement between the numerical and measured results. Figures 4(a) and 4(b) thus confirm experimentally the existence of the complete band gap located between 114 and $145 \mathrm{kHz}$.
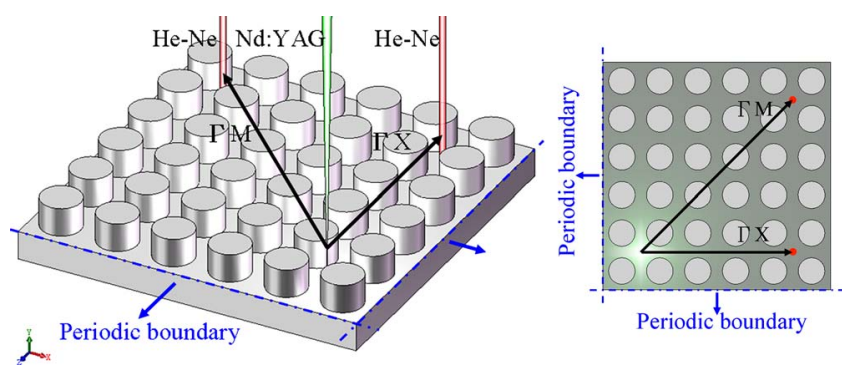

FIG. 3. (Color online) Schematic diagrams of the experimental setup. Measurements are along the $\Gamma X$ and $\Gamma M$ directions. Source and receiver are four rows apart along the $\Gamma X$ direction.
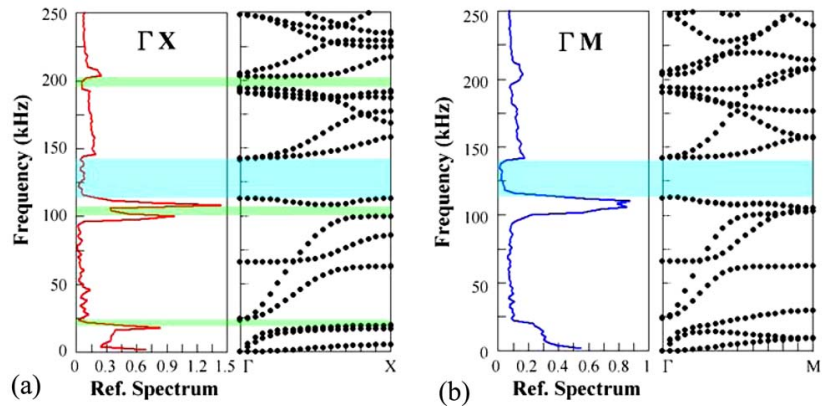

FIG. 4. (Color online) Measured reference spectra (normal displacement) for waves propagating along the (a) $\Gamma X$ and (b) $\Gamma M$ directions, respectively.

To measure both the normal and transverse displacement components, we used point piezoelectric transducers (the contact point is about $1.5 \mathrm{~mm}$ in diameter) with different polarizations. ${ }^{17}$ In the measurements along the $\Gamma X$ direction, the measured point is located at the center of the cylindrical stub and is six rows apart from the pulsed laser source. The

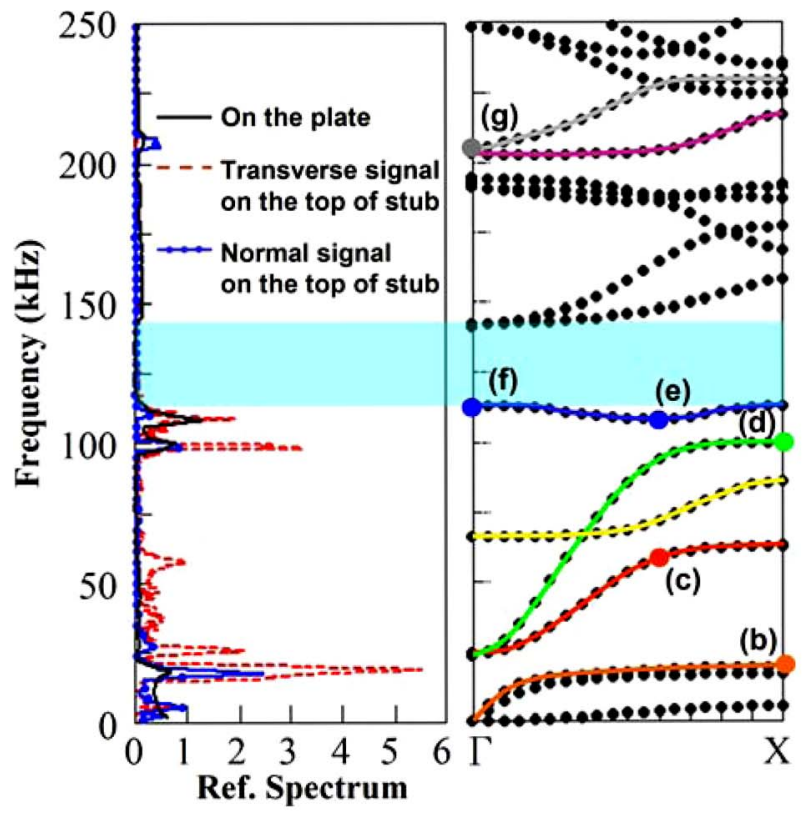

(a)

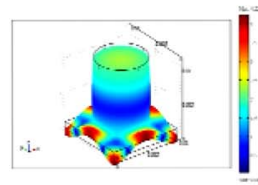

(b)

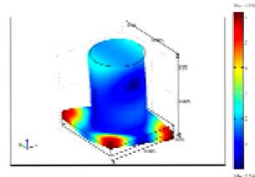

(e)

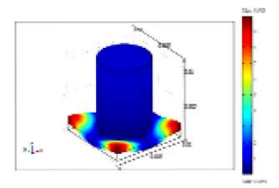

(c)

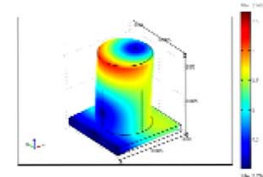

(f)

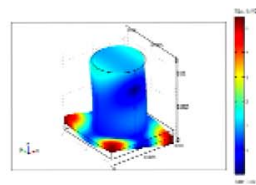

(d)

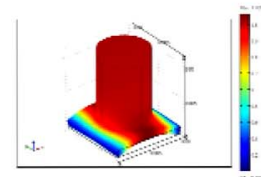

(g)
FIG. 5. (Color online) (a) Reference spectra measured on the top of the stub (both normal and transverse displacements by using point piezoelectric transducers). (b) Eigenmode analysis by FE method, with $k_{x} a / \pi=1, f$ $=19 \mathrm{kHz}$, (c) $k_{x} a / \pi=0.6, f=60 \mathrm{kHz}$, (d) $k_{x} a / \pi=1, f=100 \mathrm{kHz}$, (e) $k_{x} a / \pi=0.6, f=109 \mathrm{kHz}$, (f) $k_{x} a / \pi=0, f=114 \mathrm{kHz}$, and (g) $k_{x} a / \pi=0, f$ $=205 \mathrm{kHz}$. 
polarization of the transverse displacement measured is parallel to the wave propagation.

Figure 5(a) shows the measured reference spectra using both the normally and transversely polarized piezoelectric transducers. The measured results show that the complete band gap is coincident with that in Fig. 4(a), i.e., from 114 to $145 \mathrm{kHz}$ (blue region). For the transverse displacement (red dashed line), several peaks with values larger than one are found. Figures 5(b) $-5(\mathrm{~g})$ show the corresponding eigenmodes of the peak frequencies shown in Fig. 5(a). In each figure, the total displacement is normalized by its own maximum value.

The lowest outstanding peak is around $19 \mathrm{kHz}$, which demonstrates a notably enhanced transverse and normal vibration. Figure 5(b) shows its corresponding eigenmode, which is derived from the coupling of the $S_{0}$ and $A_{0}$ modes. For this nearly flat mode at a large wave number, resonance forms by concentrating energy in the stubs and results in a very small group velocity. The second peak, at $25 \mathrm{kHz}$, corresponds to the cutoff frequency of the modes marked with red line and green line. The third peak, appearing at $60 \mathrm{kHz}$ [point (c) in Fig. 5(a)], is governed mainly by the antisymmetric mode $\left(A_{1}\right)$, and its eigenmode is shown in Fig. 5(c). The fourth peak, appearing around $100 \mathrm{kHz}$, is related to point (d) on the mode marked with a green line in Fig. 5(a). The corresponding eigenmode, governed mainly by the $S_{0}$ and $A_{1}$ modes, is shown in Fig. 5(d). From Fig. 5(a), we find that the right-hand side of the mode (green line) is nearly flat, which results in a strong vibration in the transverse direction.

Adjacent to the peak at $100 \mathrm{kHz}$, the peak at $109 \mathrm{kHz}$ also results from the mode governed by the $S_{0}$ and $A_{1}$ modes. As shown in Fig. 5(e), a powerful vibration of the plate trembles obviously with the high stubs in the transverse direction. We note that the mode around the cutoff frequency on the left-hand side (blue line) is governed by $A_{1}$ mode only. As shown in Fig. 5(f), this mode generates vanishing transverse displacement on the top surface of the stub.

In the frequency between 143 and $193 \mathrm{kHz}$, the reference spectrum has a value about 0.15 , which means that elastic waves may propagate within this frequency range, but with perceptible attenuation. One may note that there are two nearly flat modes around $190 \mathrm{kHz}$; nevertheless, they are mainly governed by the $T_{0}$ mode (polarization perpendicular to the sagittal plane) and result in no peak in the measured spectrum.

For frequencies close to $205 \mathrm{kHz}$, the band structure exhibits two different modes. The mode marked with a purple line is mainly governed by the $T_{0}$ mode and may not be effectively generated by the laser source. Therefore, the relatively small peak appearing around $205 \mathrm{kHz}$ for normal vibration [Figs. 4(a) and 5(a)] is caused by the mode marked with a gray line. The mode is evolved from the $A_{1}$ mode and its eigenmode is shown in Fig. 5(g).

In summary, we have demonstrated the existence of the complete band gap and resonances in a plate with a periodic stubbed surface. When the height of the stubs is triple the plate thickness, a complete band gap is formed. Results of the laser ultrasonic experiments clearly show that the complete band gap and the resonances of the stubs are in very good agreement with numerical predictions.

The authors gratefully acknowledge the financial support from the National Science Council of Taiwan, Grant No. NSC 96-2221-E-002-206-MY3.

${ }^{1}$ M. S. Kushwaha, P. Halevi, L. Dobrzynski, and B. Djafari-Rouhani, Phys. Rev. Lett. 71, 2022 (1993).

${ }^{2}$ R. Sainidou, B. Djafari-Rouhani, and J. O. Vasseur, Phys. Rev. B 77, 094304 (2008).

${ }^{3}$ Y. Tanaka and S.-I. Tamura, Phys. Rev. B 58, 7958 (1998).

${ }^{4}$ F. Meseguer, M. Holgado, D. Caballero, N. Benaches, J. Sánchez-Dehesa, C. López, and J. Llinares, Phys. Rev. B 59, 12169 (1999).

${ }^{5}$ T.-T. Wu, Z.-G. Huang, and S. Lin, Phys. Rev. B 69, 094301 (2004).

${ }^{6}$ J.-C. Hsu and T.-T. Wu, Phys. Rev. B 74, 144303 (2006).

${ }^{7}$ J. O. Vasseur, P. A. Deymier, B. Djafari-Rouhani, Y. Pennec, and A.-C. Hladky-Hennion, Phys. Rev. B 77, 085415 (2008).

${ }^{8}$ F. Hsiao, A. Khelif, H. Moubchir, A. Choujaa, C. Chen, and V. Laude, Phys. Rev. E 76, 056601 (2007).

${ }^{9}$ J. O. Vasseur, P. A. Deymier, B. Chenni, B. Djafari-Rouhani, L. Dobrzynski, and D. Prevost, Phys. Rev. Lett. 86, 3012 (2001).

${ }^{10}$ B. Bonello, C. Charles, and F. Ganot, Appl. Phys. Lett. 90, 021909 (2007).

${ }^{11}$ B. Morvan, A. Hladky-Hennion, D. Leduc, and J. Izbicli, J. Appl. Phys. 101, 114906 (2007)

${ }^{12}$ COMSOL Multiphysics, Structural Mechanics, Manual, Comsol, AB, Stockholm, Sweden.

${ }^{13}$ We use the elastic constants $C_{11}=11.1, C_{12}=6.1, C_{44}=2.5$ (in units of GPa) and $\rho=2695 \mathrm{~kg} / \mathrm{m}^{3}$ for aluminium 6061 .

${ }^{14}$ T.-T. Wu and Z.-G. Huang, Phys. Rev. B 70, 214304 (2004).

${ }^{15}$ G. Wang, X. Wen, J. Wen, L. Shao, and Y. Liu, Phys. Rev. Lett. 93, 154302 (2004).

${ }^{16}$ T.-T. Wu and Y.-H. Liu, Ultrasonics 37, 23 (1999).

${ }^{17}$ T.-T. Wu and J.-S. Fang, J. Acoust. Soc. Am. 101, 330 (1997). 